
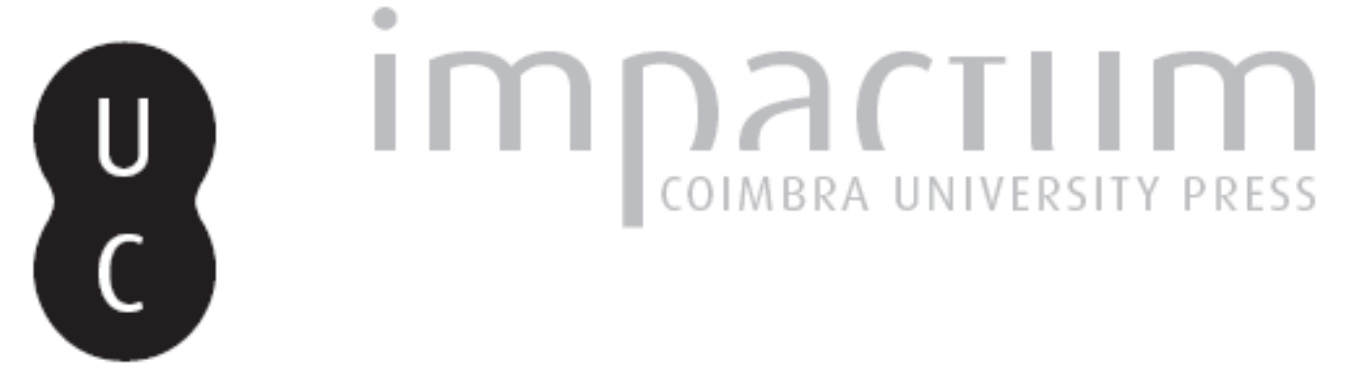

\title{
Los riesgos de incendios forestales en la zona mediterranea de Chile: un caso de perturbacion ambiental permanente
}
Autor(es):
Quintanilla, Víctor
Publicado por: Associação Portuguesa de Riscos, Prevenção e Segurança
URL persistente:
URI:http://hdl.handle.net/10316.2/36158
DOI:
DOI:http://dx.doi.org/10.14195/1647-7723_16_14
Accessed : $\quad$ 26-Apr-2023 11:50:09

A navegação consulta e descarregamento dos títulos inseridos nas Bibliotecas Digitais UC Digitalis, UC Pombalina e UC Impactum, pressupõem a aceitação plena e sem reservas dos Termos e Condições de Uso destas Bibliotecas Digitais, disponíveis em https://digitalis.uc.pt/pt-pt/termos.

Conforme exposto nos referidos Termos e Condições de Uso, o descarregamento de títulos de acesso restrito requer uma licença válida de autorização devendo o utilizador aceder ao(s) documento(s) a partir de um endereço de IP da instituição detentora da supramencionada licença.

Ao utilizador é apenas permitido o descarregamento para uso pessoal, pelo que o emprego do(s) título(s) descarregado(s) para outro fim, designadamente comercial, carece de autorização do respetivo autor ou editor da obra.

Na medida em que todas as obras da UC Digitalis se encontram protegidas pelo Código do Direito de Autor e Direitos Conexos e demais legislação aplicável, toda a cópia, parcial ou total, deste documento, nos casos em que é legalmente admitida, deverá conter ou fazer-se acompanhar por este aviso.

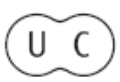




\section{territorium}

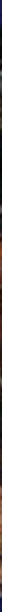

16

Revista da Associação Portuguesa de Riscos, Prevenção e Segurança 2009 
territorium 16

\title{
LOS RIESGOS DE INCENDIOS FORESTALES EN LA ZONA MEDITERRANEA DE CHILE: UN CASO DE PERTURBACION AMBIENTAL PERMANENTE*
}

\author{
Victor Quintanilla \\ Departamento Ingeniería Geográfica \\ Universidad Santiago de Chile (USACH) \\ Proyecto FONDECYT 1095048 \\ victor.quintanillaausach.cl
}

\section{RESUMEN}

La zona mediterránea de chile se localiza aproximadamente entre los $31^{\circ}$ y $38^{\circ}$ latitud sur, y en ella se concentra la mayor parte de la población del país. Por esta presión humana y también por el clima seco, de solo cuatro meses de lluvia y el resto del año cálido y seco, se dan condiciones favorables para la generación de fuegos forestales. Un bosque nativo específico de esta zona es el basque esclerófilo. Se analiza un sector de colinas costeras situadb alrededor de los $33^{\circ}$ latitud sur, donde existe un basque esclerófilo de tipo húmedo, junto al cual vive la palma más austral del mundb y endémica de Chile - Jibaea chilensis (Mbl.) Baillon. Este relieve ha sido afectado desde 1962 por reiteradas impactos de incendios de vegetación. Esto ha alterado la regeneración y permanencia de este bosque húmedo en Chile central, como igualmente van poniendo en peligro el hábitat de la palme chile.

Palabras claves: Bosque esclerófilo, fuegos, Jabaea chilensis.

\section{RESUMO}

A zona mediterrânea do Chile localiza-se aproximadamente entre os 31ํe e $38^{\circ}$ de latitude sul, nela se encontrando a maior parte da população do país. Por esta pressão humana e também pelo clima seco, de apenas quatro meses de chuva e o resto db ano quente e seco, verificam-se condições favoráveis à ocorrência de incêndios florestais. Unboosque nativo específico desta zona é o bosque esclerófilo. Analisa-se um sector de colinas costeiras situado à volta das $33^{\circ}$ de latitude sul, ande existe umbasque esclerófilo de tipo húmido, junto ao qual vive a palmeira mais austral do mundb e endémica do Chile - Jibaea chilensis (Nbl.) Baillon. Este relevo foi afectadb desde 1962 por reiterados impactos de incêndios florestais. Isto alterou a regeneração e permanência deste basque húmido no chile central, como igualmente vai pondo em perigo o habitat da palmeira chilena.

Palavras chave: Bosque esclerófilo, incêndios, Jabaea chilensis.

\section{RÉSUMÉ}

La zone méditerranéerne du chili est située entre les $31^{\circ}$ et les $38^{\circ}$ de latitude sud et concentre la plupart de la population du pays. La pression humine et le climat sec, avec quatre mois de pluie et huit mois de chaleur et sècheresse donnent les conditions favorables au déclenchement des feux de foret. Nous présentons une étude sur les risques du feu sur la forêt sclérqphylle humide, dans un secteur de coll ines côtières au chili méditerranéen. Cet aire est très intéressante car ici il y a des arbres de grand valeur gédbotanique, accompagnées de la palme plus australe du monde - Jibaea chilensis (Mbl.) Baillon. En plus, des incendies d'été, très fréquents depuis 1962, modifient la régénération et permanence de cette foret humide du chili central et, en même temps, met en risque I'habitat de la palme chiliene.

Mbts-clé: forét sclérqphylle, feu, Jibaea chilensis.

\footnotetext{
* Commicação apresentada ao V Encontro Nacional e I Congresso Intemacional de Riscos.
} 


\section{ABSTRACT}

Title: The risk of forest fires in the mediterranean zone of chile- a case of permanent enviromental perturbation.

The mediterranean zone of chile is located approximately between $31^{\circ}$ and $38^{\circ}$ south latitude and it concentrates the country's largest population. Because of this human pressure as well as because of the dry weather, with only tar months of rain and the rest of the year warm and dry, the conditions are favarable for the generation of forest fires. A specific native forest of this zone is the sclergphyll forest. A sector of coastal hills located about $33^{\circ}$ sath latitude is analyzed where there is a sclergphyll humid type forest next to which lives the world's sathemmost palm tree that is endemic to chile - Jabaea chilensis (Mbl.) Baillon. This relief has been affected since 1962 by repeated impacts of vegetation fire. This has altered the regeneration and permenence of this humid forest in central chile, and at the same time is putting at risk the habitat of the chilean palm tree.

Key words: Sclenphyll forest, fires, Jibaea dillensis.

\section{Introducción}

La alteración y roturación de los bosques mediterráneas del área de estudio, localizada en la zona más poblada del país, se inició hace 4 siglos. En este proceso, los incendios forestales tuvieron un efecto devastadbr en la el iminación y transformación de los ecosistemas, y muy en particular sobre el basque esclerófil lo de llanos y medias laderas, y en menor grado en los bosques de fagáceas de las cordilleras andina y costera.

Estudiamos un área de colinas costeras de chile central, lugar donde se desarrollan residuos de bosques esclerófilos de ambientes húmedos, los cuales conviven con la palma más austral del mundo y endémica de Chile (Jibara chilensis (Mbl.) Baillon).

Ios ecosistemas litorales han sidb afectados por fuegos regulares de verano desde hace unos 45 años, afectando y alterando peligrosamente la regeneración de estos bosques, y my en particular a urbaea diilensis.

\section{abjetivos}

Los objetivos principales que tuvo esta investigaciónfueron:

- Estudiar el alto gradb de conlaustibilidad vegetal de un área costera de chile central mediterráneo ( $32^{\circ}$ $\left.40^{\prime}-33^{\circ} 10^{\prime} \mathrm{Sur}\right)$, en la cual los fuegos vegetales de verano han sido frecuentes desde hace alrededor de medio siglo.

- Procurar determinar el grado de degradación actual del bosque esclerófilo húmedo, que se desarrolla fundamentalmente en colinas con exposicióna la influencia marina del océano Pacífico.

- Determinar las perturbaciones a que está expuesta la palma más austral del mundo Jubaea chilensis (Mbl.) Baillon por estos rearrentes fuegos; por cuanto dicha palmácea comparte aquí su hábitat con el bosque esclerófilo.

\section{Área de estudio}

El sector litoral de col inas frecuentenente afectada por fuegos vegetales estivales, se localiza al interior de Chile mediterráneo, aproximadamente entre las coordenadas geográficas $32^{\circ} 42^{\prime}$ y $33^{\circ} 48^{\prime}$ latitud sur (QMNIAIIA, 1998; CAsTHIO, 2006) (fig. 1) .

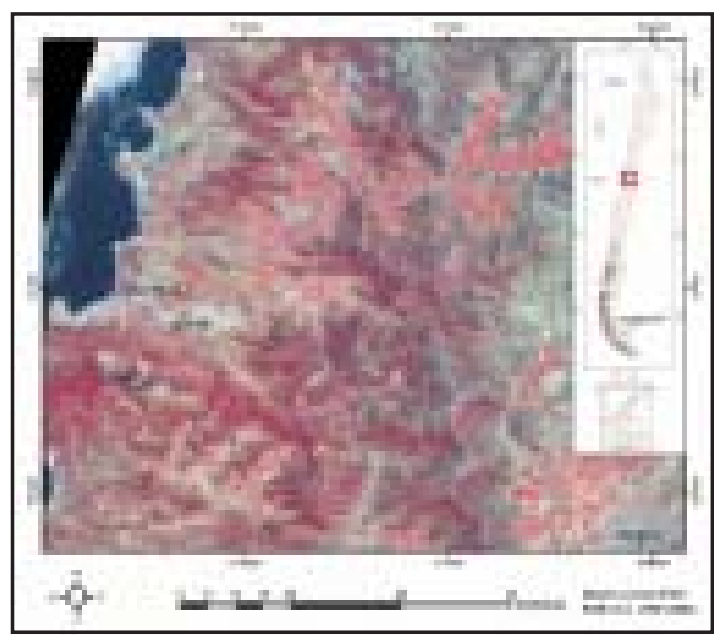

Fig. 1 - Localización del área de estudio en chile Mediterráneo.

Este relieve margina una importante conurbación urbana compuesta por las ciudades de Valparaíso, Viña del Mar y Quilpué; las cuales alcanzan una pablación aproximada de 1.000.000 de habitantes. Las cotas más altas de estas colinas alcanzan los 770 m.s.n.m. , disectadas por numerosas quebradas de corta extensión que se desplazan con valores de pendientes entre los 35 y 15 grados (fig. 2) .

Los suelos predaminantes son principalmente de tipo granítico, con afloramientos rocosos dispersos en sectores de mayor pendiente, y presencia de material arcill loso, sobre el aal se doservan a menubb procesos erosivos hídricos freauentes, e inclusive, conpresencia de formación de cárcavas (QunNantia, 1998) . 


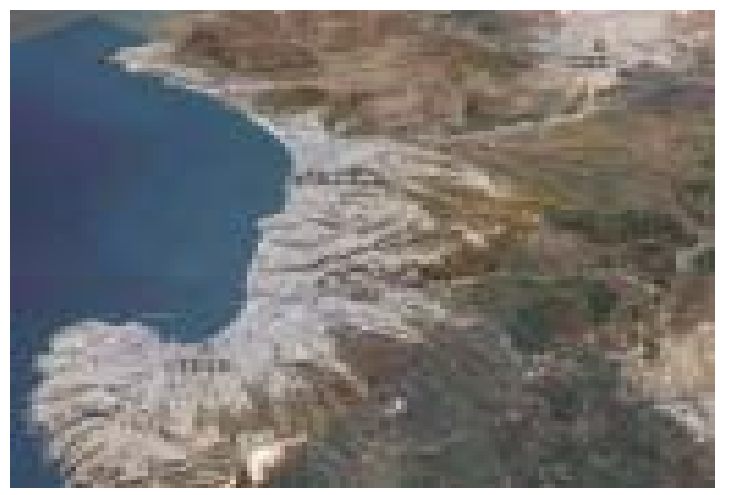

Fig. 2 - Panorámica del área de estudio. Limite del sector urbano bajo la influencia marina (Google Earth, 2009) .

Ell clima de la franja costera presenta de manera my diferenciada las cuatro estaciones, con lluvias concentradas en otoño e inviemo que no superan los 400 mmanales (fig. 3) . Ell régimen térmico se caracteriza por una temperatura media anul de $14,8^{\circ} \mathrm{C}$. La estación seca comienza en noviembre y termina en abril, o sea dura 7 meses (NboA et al., 1989).

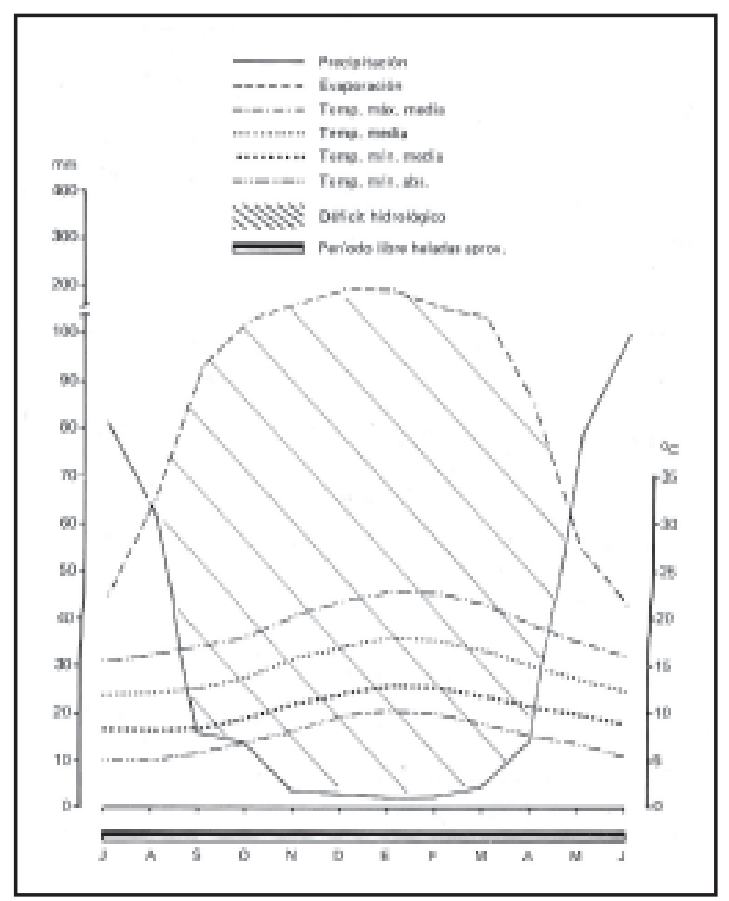

Fig. 3 - Climodiagrama de Valparaíso $\left(33^{\circ} 01^{\prime} \mathrm{W}-71^{\circ} 39^{\prime} \mathrm{S}\right)$.

Es importante destacar que en este sector el litoral tiende a conformar una morfología con bahías relativamente cerradas, las cales concentran una gran cantidad de nieblas matinales, inclusive durante el verano. Esta humedad atmosférica es muy importante para permitir la permanencia de las agrupaciones vegetales de tendencia húmeda en la costa de la región.

Asimismo la vegetación hoy en día muestra testimonios diversos de variadas comunidades, a pesar de la fuerte presión antrópica a la cual ha estado expuesta desde hace más de medio siglo. De acuerdo con VIIIASEÑOR (1977) y QUINIANUIA (1998), puede encontrarse un matorral xerófilo como matorral secundario en laderas asoleadas de exposición norte; agrupaciones de bromeliáceas (Pryales) que están en contacto con las anteriores; bosque esclerófilo mésico semiabierto; matorral esclerófilo abierto y bastante intervenido; basque esclerófil o húmedo, donde son dominantes árboles de umbría como Peumus bolcus, cryptocaria alba y Kageneckia oblonga; y en laderas bajas, sobre todo expuestas hacia la influencia marina, encontramos las comunidades de palma chilena (Jibaea chilensis) (tot. 1, 2y3).

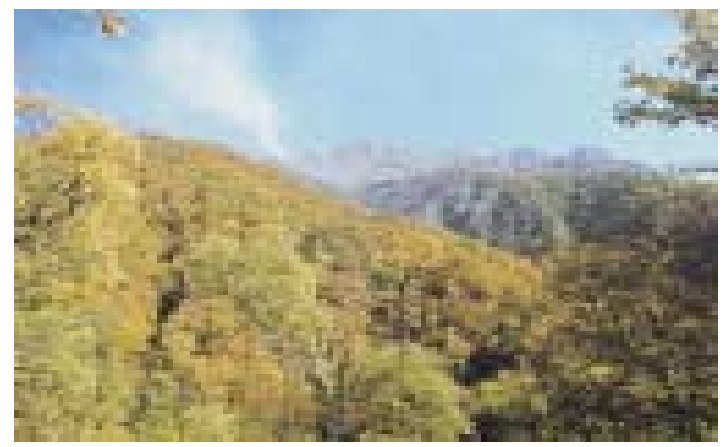

Fot. 1 - Bosque esclerófilo en chile central.

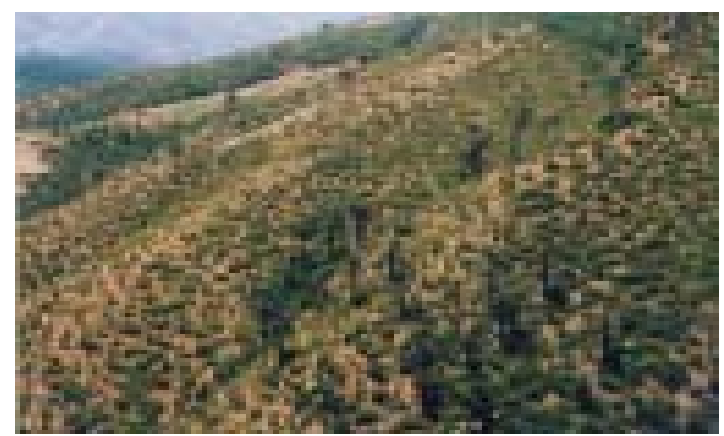

Fot. 2 - Laderas con retroceso de vegetación esclerófila e iniciación de procesas erosivos.

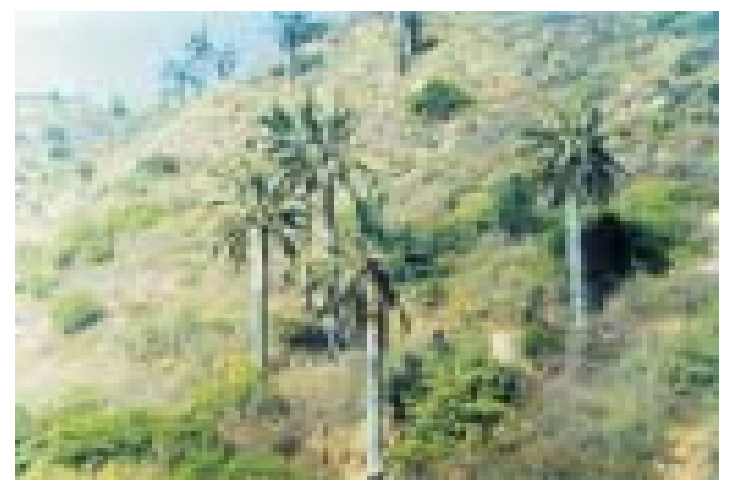

Fot. 3 - Palma dhilena (Jubaea chilensis) en matorral mediterráneo abierto. 


\section{Materiales y métodos}

En la metooblogía se siguieron fundamentalmente los pasos tradicionales de toda investigación. De este modo se analizó la documentación bibliográfica y cartográfica disponible da la zona mediterránea de Chile, utilizándose cartas topográficas (escalas 1:50.000 - 1:100.000) para los trabajos de campo. Ias estadísticas climáticas fueron revisadas en los anales de la Meteorología de chile; en tanto para los registros de fuegos se consultaron las bases estadísticas de la Corporación Nacional Forestal (CONAF) desde el año 1975.

En gabinete a su vez se analizaron distintos materiales gráficos, realizando fotointenpretación de clichés pancramáticas (escala 1:60.000) de los años 1976, 1977, 1983 y 1995; y de ortofotos (escala 1:20.000) del año 1996. También se analizaron y procesaron distintas imágenes satelitales Landsat (MSS, TM, EIM), apoyándose además de una imagen SPOT falso color del año 1987.

Ell trabajo de campo se real izó en los períodas de verano, otoño y primavera de los años 1998, 2005 y 2006. Se efectuaron muestreos fitosociológicos en parcelas de 20x20 metros; elaborando además transectas fitogeográficas.

\section{Discusión y Resultados}

Considerando que las unidades vegetacionales actualmente presentes en el área de estudio tienden a formar un gran arco periférico a la comurbación urbana de las ciudades de Valparaíso, Viña del Mar y Quilpué adquieren un alto riesgo permanente de incendios durante gran parte del año, con excepción de invierno. Se coincide con CAsIRO \& QuINIANIIIA (1998) respecto a que el riesgo de incendio equivale al peligro inminente de que determinadas circunstancias, permanentes o transitorias, permitan la ocurrencia o aparición de un fuego incontrolado que produce daños ecológicos y económicos en los ecosistemas vegetales.

De la síntesis de los resultados de los censos fitosociológicos realizados por Q QRIIAMUA (1998) Y CAswito (2006), se destaca la irregular regeneración de las especies del matorral y del bosque esclerófilo, con posterioridad a los fuegos que se produjeron ininternmpidamente entre los veranos de 1997 y 2006 en este mismo sector costero.

Se debe señalar también que en estas pequeñas montañas de antiguos hábitat de bosque nativo esclerófilo, existen actualmente al rededor de 12.000 ha de plantaciones de pino insigne y de eucal iptos. Estos últimos, por el alto poder de combustibili idad de sus ramas y hojas, son responsables en gran parte de la expansión de los numerosas fuegas del estío en el área costera (fot. 4) .

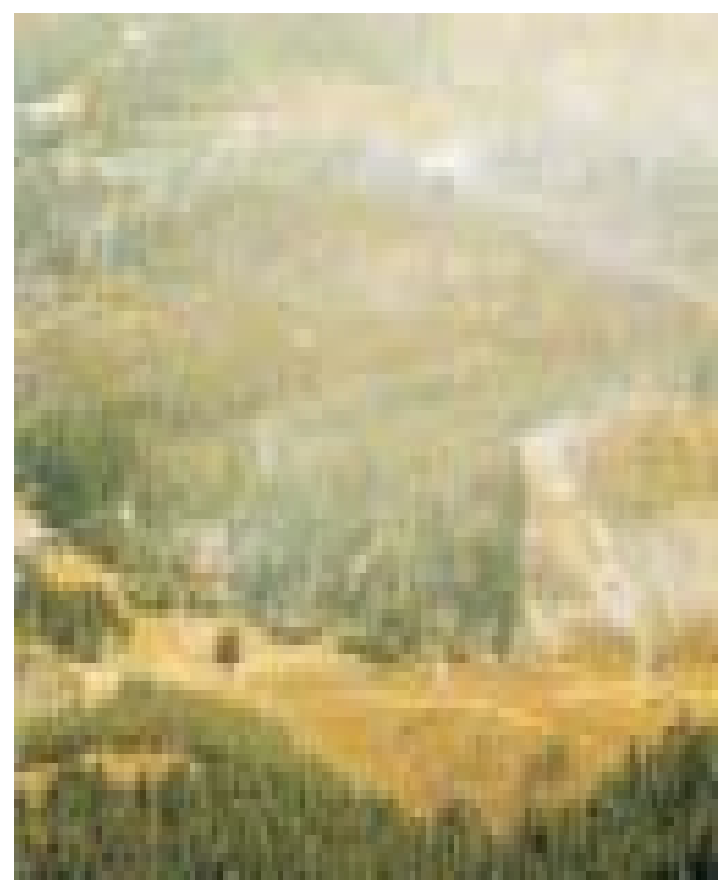

Fot. 4 - Laderas con repoblaciones forestales afectadas por los fuegos.

Ell bosque esclerófil lo húmedo y mesotérmico era típico de la zona, predominando comunidades de Cryptocaria alba, Peumus boldus, Bei Ismiedia miersi i y Qui 7 laja saponaria. En quebradas expuestas al sol, y sobre laderas de suaves pendientes o en fondo de estrechos valles, eranmás constantes las agnupaciones de Quillaja saponaria, Schinus latifolius y Lithraea caustica. Dada la estructura continua que en un principio tuvieron estos bosques, resultaron ser con el tiempo las agnupaciones más afectadas por el fuego (QUINIANMIA, 1983).

Los incendios no sólo han ido afectando la estructura y composición de las comunidades vegetales, sino que también han ido causando una importante degradación en el suelo (fot. 5) .

Se advierte la constante perdida de la cubierta vegetal del sustrato, lo cual permite que los suelos estén más susceptibles de ser erosionados por la acción de la lluvia y el viento; elementos que determinan el arrastre de los subhorizontes superficiales del suelo. Esto implica tanbién la péroida de semillas, lo cual se traduce en el retardo de la eventual regeneración de las plantas. Considerando esto último, se doserva que la composición florística de las comunidades puede variar, siendo éste un indicador importante de modificaciones del ecosistema. No obstante, y de acuerdo con Avır et al. (1988), se comprueba que en ésta área existen 


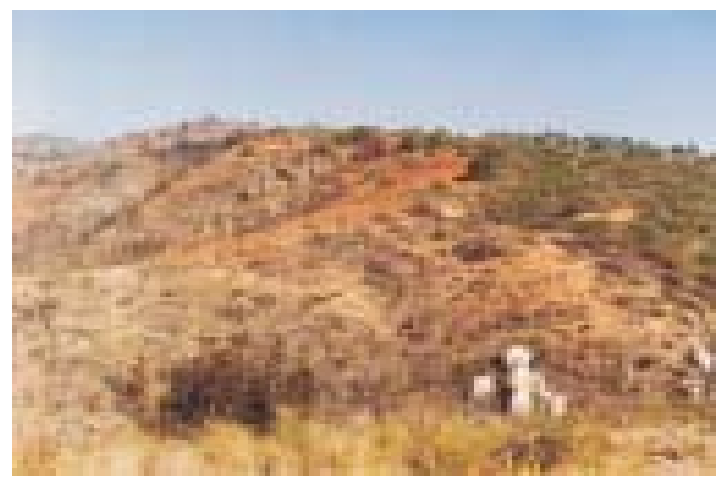

Fot. 5 - Desmonte por fuego del matorral esclerófilo, generando como consecuencia procesos erosivos.

especies denominadas pioneras como Muehlenbeckia hastulata, Baccharis rosmarinifoliay Eryngium fasciculatum, las que tienden a colonizar espacios abiertos dejadas por el paso del fuego. En aquellas laderas de quebradas, con exposición al mar, se doservó que esta colonización es más activa, e incluso suele desarrollarse una bambúcea (Chusquea amingii) .

Debido a las propiedades morfológicas y fisiológicas de las plantas, apoyadas por la humedad costera, vuelven a reestablecerse plantas postfuegos. Ias formas de recuperación pueden ser a través de la germinación de semillas en lugares quemados o a través del desarrollo de yemas que no han sido afectadas por el fuego. Badduzi et al. (1981) comprobaron que la recuperación de yemas puede darse porque se encuentranbajo el suelo en las hierbas perennes y algunos arburtos, o bien se hallan protegidas del fuego por la corteza del árbol o arjusto.

Ell rebrote experimentado en árboles y arbustos produce una rápida reaperación del follaje perdido, con renovación de los órganos de la planta y variaciones en su forma original.

Candb los individucs del estratoarbustivodesanollan brotes a partir de yemas subterráneas, se generan renovales, reproduciéndbse a través de unamitiplicidad de tallos que energen simultáneamente (fot. 6) .

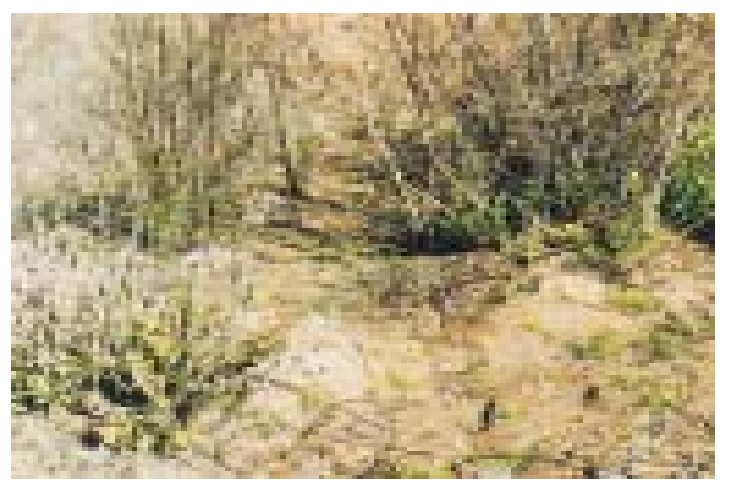

Fot. 6 - Regeneración postfuego del sotobosque del matorral esclerófilo.
A través de la realizaciónde censos fitosociológicos (40 x 40 m) efectuados en sectores que habían sido afectados por fuegas entre 1996 y 2006; se detectó una recuperación importante de árboles-arbustos nativas. Sin embargo la estructura de la agnupación ha ido variando, por cuanto encontramos presencia de plantas invasoras, incluso algunas de ellas pertenecientes a ecosistemas templados.

Fh el CAro I se destacan los valores de presencia de especies local izadas. Cabe mencionar la ausencia de la palma chilena (Jibaea chilensis) , como así también, la presencia de Rubus ulmifol ius, arbusto conuna adaptación más favorable a los medios templadbs.

Disturbios rearrentes como los incendios, son también constantes en esta zona, debidb a que las comnidades arbustivas del área son altamente inflamables en las temporadas del fuego en la zona mediterránea diilena (1 novienbre - 30 abril) ; especialmente porquemudas de ellas son deciduas de verano, y por el abundante estrato herbáceo que suele acompañarlas, particularmente arandoel inviemo ha tenidb el evadosmontos deprecipitación. Por otra parte, es reconocidb que debidb a la intervención humana, en el piedmont y montañas costeras de la zona mediterránea, predominan en la actual idad agnupaciones secundarias como resultadb de las intensas degradaciones a que fue expuesto el bosque primitivo. Fntreellas, el rol de los incendios ha sidbunode los grandes factores del desarrollo posterior de una formación que actualmente es denominada 'matorral esclerófild", yque posee una cierta semejanza con la maquia europea.

A los pocos meses de producidos los incendios, comienza el rebrote de algunas plantas, que son favorecidas adenás por las precipitaciones y las nebl inas matinales costeras. Antes de 4 meses de transamrido un fuego, se puede detectar el rebrote de Fryngium fasciaulatum, arbusto perenne que puede alcanzar unos 60 a 80 cmde alto (fot. 7). Fitre los ánboles, Lithraea caustica rebrota, a su vez, a partir del lignotuber en la producción de ramas nuevas que nacen del mismo punto, iniciándase su regeneración más o menos a los 45 días deproducidb el incendio.

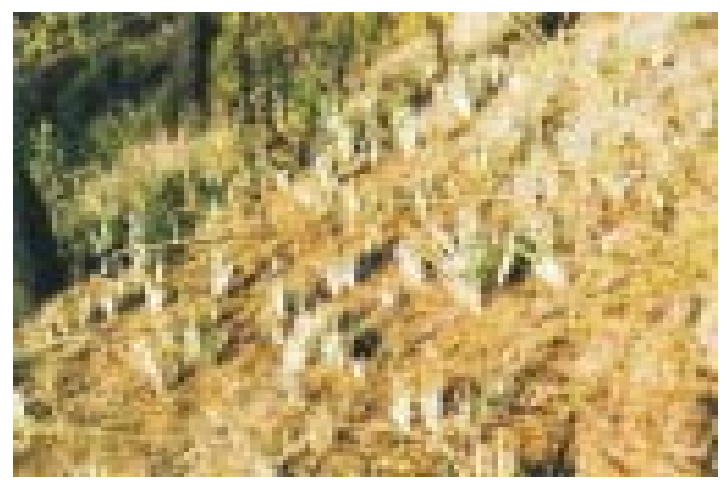

Fot. 7 - Regeneración de Eryngium fasciculatum en áreas intervenidas por los fuegas. 
CAIro I - Valores de presencia de plantas más contantes en muestras de terreno (parcela 40 m²). IX-1996 a VI-1997

\begin{tabular}{|c|c|c|c|c|c|c|c|c|c|c|c|c|c|c|c|c|c|c|c|c|c|c|}
\hline \multicolumn{23}{|l|}{ ryilithin } \\
\hline 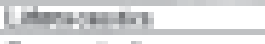 & E & I & 1 & I & II & 1 & II & F & If & I & 9 & I. & II & 1 & 4 & 1 & II & 1 & 7 & 1 & & \\
\hline Dranilly & . & & & 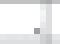 & I & J & I & 1 & I & I & $\mathbf{L}$ & & 1 & & I & & . & & &. & & I \\
\hline 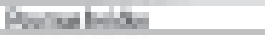 & 1 & & & $n$ & 15 & & & 1 & 7 & & & 8 & 1 & & 1 & 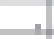 & $\Perp$ & 1 & ; & 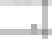 & 1 & \\
\hline 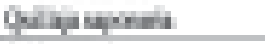 & 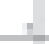 & 1 & & 1 & 7 & 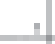 & 1 & 1 & $=$ & 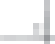 & 1 & 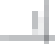 & 1 & 7 & 1 & 1 & 1 & 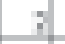 & & 1 & & F \\
\hline Hhestring & 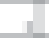 & & & I & I & & & & & | & 7 & 1 & 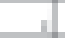 & & 1 & 1 & & 1 & & 1 & & 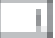 \\
\hline Itwathith & - & & & & & & & I & $=$ & 1 & $=$ & & & & & & & & & 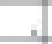 & & \\
\hline Drntalshil & . & 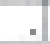 & & . & &. &. & 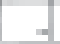 &. &. & . & & . & 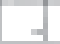 &. & . &. & 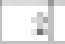 & & 1 & I & I \\
\hline \multicolumn{23}{|c|}{ 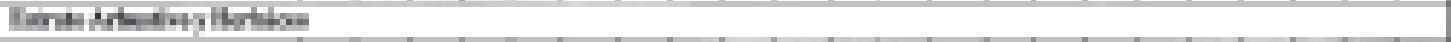 } \\
\hline beptintip & . & . & 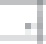 & 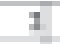 & & 1] & 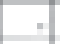 & + & 4 & $\Rightarrow$ & 7 & & $\cdot$ & 1 & . & J & , & I & & . & $n$ & \\
\hline 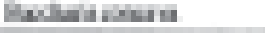 & re & 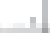 & & & & 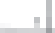 & ( & F & A. & II & II & & 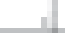 & i & in & 9 & II & A & & 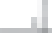 & & \\
\hline Bathriemanth & 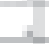 & 3 & & iI & & 1 & 1 & A & & . & 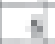 & & & H & & ) & & 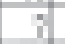 & & 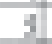 & 1 & + \\
\hline Hratumit & - & & & & H & 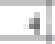 & 7 & $y$ & & H & . & s & . & H & . & 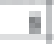 & . & m & & 4 & 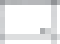 & H \\
\hline Pethelen &. & . & . & . & & . & 1 & $=$ & 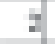 &. & 4 & It & . & t &. & $\mathbf{y}$ & . & - & H & . & & \\
\hline Tresthen & $=$ & . & 11 & 7 & & II &. & $=$ &. & - &. & 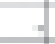 &. & $=$ &. & . &. & $=$ & $=$ & . & $=$ & \\
\hline Hemether & $=$ & 1 & 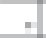 & . & & . & 1 & - & 1 & - & I & &. & - & ] & . &. & 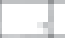 & 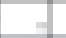 & - & 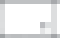 & 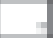 \\
\hline Micher & $=$ & , & 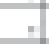 & $=$ & & 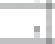 & 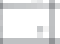 & A & 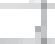 & I & 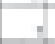 & E & 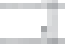 & 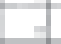 & 1 & 1 & 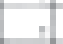 & ; & $=$ & 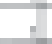 & 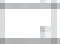 & \\
\hline 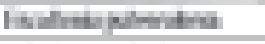 & 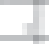 & 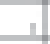 & & 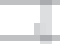 & & 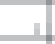 & 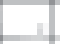 & T & 1 & 1 & 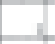 & & 7 & & 4 & 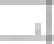 & 1 & 8 & $\mathrm{H}$ & & 1 & \\
\hline Fithopatullun & 4 & 1 & 1 & $I$ & F & 1 & 1 & A & IA & 4 & 7 & H & II & A & I & 111 & H. & 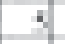 & i1 & & II & 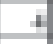 \\
\hline Furdetrin & $=$ &. & . & . & &. &. & $=$ & I &. & $\Leftrightarrow$ & I & 1 & $=$ & 1 &. & 1 & 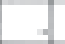 & $=$ & . & $=$ & 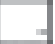 \\
\hline 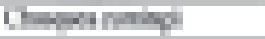 & $=$ & . & . & . & & . &. & 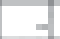 &. &. & . & $F$ & . & $=$ & ti & . & E & $=$ & $=$ & - & $=$ & 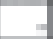 \\
\hline 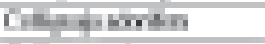 & $=$ & . &. & . & . & = & if & II & H & II & II & F & 1 & T & 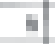 & I & 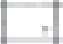 & I & 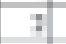 & . & $T$ & 7 \\
\hline 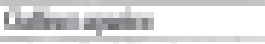 & III & 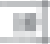 & H & 71 & I & 14 & II & 7 & 8 & a & 5 & A & 4 & $\pi$ & $\boldsymbol{H}$ & 14 & $\Pi$ & II & I & H & 11 & $\mathrm{H}$ \\
\hline \multicolumn{23}{|l|}{ Arlbhing } \\
\hline Fitrporitalu & & & & & $H$ & + & & & $=$ & H & & it & & I & . & 4 & . & + & & . & & \\
\hline 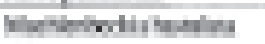 & if & I & 1 & 5 & I & . & II & $=$ & IL & . & $=$ & $\mathbf{H}$ & 7 & + & 1 & t &. & $=$ & $=$ & . & $=$ & 4 \\
\hline Lallatil & $=$ & . & . & I & & I & I & $A$ & $=$ & 7 & $=$ & 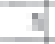 & I & 1 & J & 1 &. & 4 & $=$ & 1 & I & 1 \\
\hline 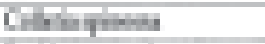 & $=$ & . & . & . & & 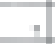 & $n$ & - & 4 & 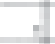 & 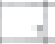 & 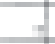 & . & $=$ & 1 & 1 & 1 & & $=$ & 1 & 1 & 1 \\
\hline hedingrily & $=$ & 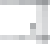 & 1 & 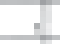 & & 1 & 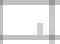 & & & $r$ & 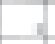 & + & 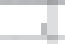 & 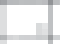 & 1 & 1 & 1 & 4 & F & i & 1 & 1 \\
\hline andring & + & 7 & 1 & 4 & i & 7 & H & III & 17 & 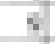 & 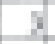 & $=$ & 19 & H & 7 & . & 1 & A & 1 & 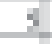 & I & H \\
\hline Fllokratrilli & & & if & & $H$ & 7 & I & a & $=$ & 1 & r & & & + & 1 & & 11 & & if & & H & H \\
\hline Frifophi & & & i & 1 & $H$ & : & it & i) & 1 & 1 & $=$ & F & 1 & 1 & 7 & $=$ & 1 & 1 & 1 & 2 & 4 & . \\
\hline Myther & & & 1 & 1 & ; & 5 & 1 & H & 5 & & 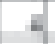 & & 4 & A & & 1 & & I. & & 4 & & \\
\hline Death inghth & & & & I & H & I & $\mu$ & I) & 7 & I & I & & 5 & 7 & 7 & F & 7 & 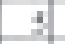 & 1 & 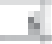 & II & H \\
\hline Ihather & 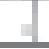 & 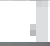 & 1 & 1 & 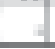 & 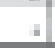 &. & I & & -1 & 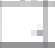 & & . &. & & 1 & & & & & & \\
\hline
\end{tabular}

Atroárbol esclerófilo con una relativa regeneración rápida en hábitats más húmedos, ha sido cryptocaria alba, aunque su rebrote se ve difiaultadb a causa de la fuerte herbivoría de liebres y conejos sobre los renuevos, e inclusive del ganado vacuno. Según VIIIASEÑ̃̃R \& SATZ (1993) , su crecimiento se acelera sólo después de la primavera, aunque de modo más lento que Lithraea cáustica. Algo similar oarriría con otro abundante árbol del bosque esclerófil o que prefiere las áreas asoleadas, como es Qui 7 laja saponaria.

Fn el estrato ardustivo sólo algunas especies han demostrado una mayor capacidad de recuperación y colonización postfuego dentro de un períodb de tiempo de unos 8 a 12 meses, como son: Podanthus mitiqui, Muhlenbedkia hastulata y Baccharis rosmarinifolia. In canbio, los arluustos queprefieren terreno més asoleadbs retardan casi un año su proceso de regeneración después de los siniestros. Se destacan entre los más representativos: Colliguaja oobrifera, Baccharis paniaulata, Adesmia phylloidaa y A. arborea.

En cuanto a la cubierta hendácea del matorral esclerófilo, ésta se recupera rápidamente después de las primeras lluvias (mayo). Sin enbargo, al cabo de estos últimos años, se han detectado cambios importantes, particularmente en el tamaño y número de individuos por unidad de superficie. Al parecer hay especies nativas que tienden a disminuir su densidad, en cambio otras, preferentemente introducidas, tienden a aumentar significativamente. Es el caso por ejemplo de: Avena barbata, Erodium cicutareum, Untica urens, Astragalus berteriamus, Matricaria recutita, Rammculus repens y Cynara cardumaulus; la mayoría de ellas consideradas como "malezas" (hierbas y gramíneas).

Esta continua pérdida y debilitamiento de la abierta vegetal, ha producidb en numerosos sectores 
de pendientes superiores al 10\%, procesos erosivos por acción de las precipitaciones y el viento. Tal fenómeno implica además la pérdida de semillas en ese sueloy que se refleja en el retardb de la eventual regeneración del ecosistema (fot. 8) .

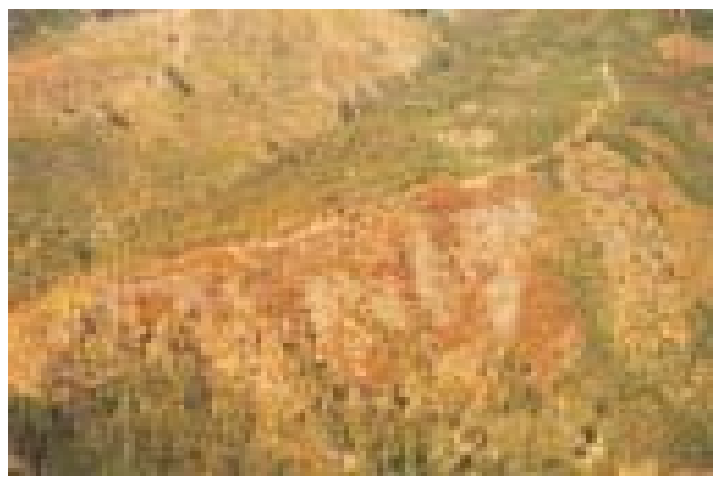

Fot. 8 - Procesos de erosión en laderas de fuertes pendientes afectadas por los incendios.

Otro factor de incendio en las plantas del matorral, es la manutención de la actividad de las hojas por períodas de 1 o 2 años, excepcionalmente 304 ; lo que permite la existencia de un permenente estrato de hojas caídas con diferentes grados de descomposición. A esto se suma la existencia de plantas que pierden sus ramas completas, trozos de corteza y otros elementos, ayo conjunto constituye un material conbustible susceptible de ser quemadb. Estos factores se ven incrementados por la presencia de otras características que acentúan el riesgo de fuegos vegetales en el área. Por ejemplo, como lo constató AmIA (1988) , la existencia de resinas, aceites esenciales, pelos, espinas, unbajo contenido de agua y las gruesas atíaulas presentes en las hojas de las plantas dominantes, favorecen la combustión.

Por último, los elementos climáticas son otros de los factores responsables del constante riesgo de fuegos en este litoral debajos relieves. Yactros autores se habían referido a este tema de los procesos meteorológicos, asociadbs con los índices de riesgo de incendios en zonas mediterráneas (Juro 1993; Miria et al. , 1998).

Los vientas provenientes del suodweste durante primavera y verano, llegan con mucha frecuencia a la costa mediterránea de Chile, constituyéndbse de este modo en uno de los elementos indispensables para la ignición de la vegetación.

\section{Conclusión}

Los riesgos de fuegos en la vegetación de tipo esclerófil la del cordón costero del área de estudio en chile mediterráneo son permanentes y los incendios se suceden todbs los veranos y principios de otoño.
Considerando la irregularidad climática que también se manifiesta en toob el territorio chileno en estos últimos años, se puede esperar que estos siniestros próximamente se inicien a comienzos de primavera, antes de la temporada oficial, considerada en chile para estar alerta a los incendios forestales (novienbre-abril) .

Por otra parte se doserva un enrarecimiento de las commidades en situaciones post-incendios, al introducirse en los ecosistemas quemados, plantas pioneras de rápido crecimiento (arbustivas y herbáceas) , y que también van transformando la estructura del matorral esclerófilo, el que tiende en estos momentos, a crecer de forma fragmentada y marginada en los sectores más elevados de las colinas, por repoblaciones de Pinus sylvestris y Encaliptus gldailus.

Se coincide con CAsiwro (2006) que en el área de estudio se detectan patrones de movilidad tanto en la oarrencia de incendios como en la extensión de éstos. A raíz del crecimiento urbano de las ciudades de Valparaíso, Viña del Mar y Quilpué, las poblaciones están ocupando terrenos en la parte baja de las colinas. Esto ha incidido que varios focos de fuegos se inicien en estos lugares, muy próximos a las agnupaciones esclerófilas, y en cuya periferia ya paseen varias especies alóctonas.

Por otra parte esta situación de peligro de combustión, se acrecienta también para los ejemplares de la palma chilena (Jibaea chilensis) .

\section{Referencias Bibliográficas}

Araya, S \& AvIA, G. (1981) - "Rebrote de arbustos afectados por el fuego en el matorral chileno". Anales Museo Historia Natural, 14. Santiago, p 107-113.

Avila, G. ; Aluaro, M. ; Montenegro, G. (1988) "Incendios en la vegetación mediterránea de Chile". Fr: Fuentes, E. \& Prenafreta (eds) Ecología del Paisaje de Chile Central. Universidad Católica de Chile. Santiago. p 81-86.

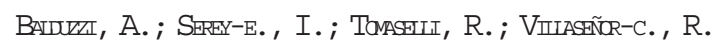
(1981) - . "New phytasociological observations on the Mediterranean type of climax vegetation of central Chile". Atti Ist. Bot. Uhiv. Iab. Critt. Pavia, serie 6, 14, p.93-112.

CAstuto, M. (2006) - El cambio del paisaje vegetal afectado por incendios en la Zona Mediterránea Castera de la V Región. Tesis para optar al Grado de Magíster en Geografía. Universidad de chile. $155 \mathrm{p}$.

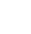


CASIRO, R. \& QUNIANIIA, V. (1998) - Seguimiento de abiertas vegetales postincendios forestales en la zona mediterránea costera de Chile. Serie geográfica, Vol. 7. Depto de Geografía. Universidad de Alcalá de Henares. España.

Juro, G. (1995) - Actas del Taller Intemacional Prognosis y Gestión en control de Incendios Forestales. Proyecto FONDEF FL-13 CONICYT. . Universidad de Chile-INFOR-INIEC. Santiago p 68-89.

MEJÁN, M. M. ; EsTREIA, M. J. ; BADENAS, C. (1998) 'Meteorological processes relevant to forest fire dynamics on the Spanish Mediterranean coast" . Joumal of Applied Meteorology, 37, p. 83-100.
NovoA, R. \& VIIAAsca, S. (1989) - Mapa Agroclimático de Chile. Fdiciones Instituto de Investigaciones Agrarias, INIA, Ministerio de Agrialtura. 221 pp.

QuINAMIIA, V. (1998) - Los incendios de vegetación en el cordón costero de chille Central. El apoyo de la cartografía para su gestión en la prevención y análisis. Editor Universidad de Santiago de chile. 75 p. Carta a color. Santiago. Chile

VIrAANTR, R. \& SÁm, F. (1993) - "Incendios forestales en el Parque Nacional La Campana Sector Ocoa, VRegión, chile. Efecto sdore el estrato arbustivoarbóreo" . Anales Museo de Historia Natural de valparaíso, chile. 21, p.15-26. 